\title{
Desarrollo de una función de perfil mediante modelos mixtos para Pinus sylvestris en Turquía: selección de parámetros fijos a expandir
}

\author{
Development of a stem taper function using mixed-effects models for Pinus sylvestris in Turkey: \\ selection of fixed parameters to expand
}

\author{
Esteban Gómez-García ${ }^{a}$, Ulises Diéguez-Aranda ${ }^{\text {b }}$, Ramazan Özçelik ${ }^{\text {c }}$, Marcos Sal-Cando ${ }^{\text {b }}$ \\ Fernando Castedo-Dorado ${ }^{d}$, Felipe Crecente-Campo ${ }^{\text {, }}$, José Javier Corral-Rivas ${ }^{\mathrm{f}}$, Manuel Arias-Rodil ${ }^{\mathrm{b}}$ * \\ ${ }^{a}$ Centro de Investigación Forestal de Lourizán, Xunta de Galicia, Pontevedra, España. \\ *Autor de correspondencia: ${ }^{b}$ Universidad de Santiago de Compostela, Departamento de Ingeniería Agroforestal, Unidad de Gestión \\ Forestal Sostenible (UXFS), Escuela Politécnica Superior, C/ Benigno Ledo, 27002, Lugo, España, manuel.arias.rodil@gmail.com \\ c Süleyman Demirel University, Faculty of Forestry, Isparta, Turquía. \\ ${ }^{d}$ Universidad de León, Departamento de Ingeniería y Ciencias Agrarias, Ponferrada, España. \\ ${ }^{\text {e } C E R N A ~ I n g e n i e r i ́ a ~ y ~ A s e s o r i ́ a ~ M e d i o a m b i e n t a l ~ S . L . P . ~ L u g o, ~ E s p a n ̃ a . ~}$ \\ ${ }^{\mathrm{f}}$ Universidad Juárez del Estado de Durango, Facultad de Ciencias Forestales, Durango, México.
}

SUMMARY

Stem taper functions predict diameter variation along the tree stem, enabling to estimate merchantable volume according to market requirements. In this study, a stem taper function for Pinus sylvestris in Turkey was developed using the mixed-effects modelling approach, which allows some parameters to include a fixed part (common to the whole population) and a random effect (individualspecific response). We analyzed different strategies to choose the best combination of fixed parameters to expand with random effects (mixed-effects parameters): (1) expanding fixed parameters that presented the highest variability and (2) all possible combinations of one and two mixed-effects parameters; the best performance was observed in the latter. Given that inclusion of random effects was not enough to account for the existing correlation between the residuals of the same individual, the variance-covariance matrix of the error term was modelled by a first-order autoregressive structure. In addition, we evaluated the response obtained by calibration (estimation of random effects for a new individual), i.e. using a diameter measured at different heights along the stem. The selected mixed-effects model presented the best results both in the fitting and calibration steps. Generally, the mixed-effects model is recommended if an additional stem diameter measurement at $40-90 \%$ of the total tree height is available. Otherwise, and from a predictive point of view, the model fitted by non-linear ordinary least squares is recommended, which only considers fixed parameters.

Key words: Max and Burkhart, autocorrelation, calibration.

\section{RESUMEN}

Las funciones de perfil predicen la variación del diámetro a lo largo del tronco del árbol, y se emplean, principalmente, para desarrollar tarifas de cubicación con clasificación de productos. En este trabajo se desarrolló una función de perfil para Pinus sylvestris en Turquía empleando la metodología de modelos mixtos, donde algunos parámetros del modelo incluyen una parte fija (común a toda la población) y un efecto aleatorio (específico para cada individuo). Se analizaron diferentes estrategias para seleccionar la mejor combinación de parámetros fijos a expandir con efectos aleatorios (parámetros de efectos mixtos): (1) aquellos parámetros fijos que presentaron mayor variabilidad y (2) todas las posibles combinaciones de uno y dos parámetros de efectos mixtos, resultando esta última más adecuada. Dado que la inclusión de efectos aleatorios no fue suficiente para considerar la autocorrelación de residuos, se modelizó la matriz de varianza-covarianza del término de error mediante una estructura autorregresiva de primer orden. También se evaluó la respuesta calibrada (estimación de los efectos aleatorios para un nuevo individuo) utilizando un diámetro medido a distintas alturas a lo largo del tronco. El modelo mixto seleccionado presentó los mejores resultados en las fases de ajuste y de calibración. En general, se recomienda emplear el modelo mixto si se dispone de una medición adicional del diámetro del tronco a una altura de entre $40-90 \%$ de la altura total del árbol. En caso contrario, desde un punto de vista predictivo resulta más conveniente emplear el modelo ajustado por mínimos cuadrados ordinarios.

Palabras clave: Max y Burkhart, autocorrelación, calibración. 


\section{INTRODUCCIÓN}

La planificación de la gestión forestal en Turquía tiene sus orígenes en la década de 1960. Los planes de gestión, que normalmente se aplican a una o dos cuencas hidrográficas, tienen una duración de diez años. El enfoque clásico de estos planes se concentraba en la silvicultura y la utilización de los recursos madereros, por lo tanto, los planes intentaban conseguir la máxima producción de madera y relegaban a un lugar secundario otros usos del bosque. Sin embargo, influenciados por el Programa Forestal Nacional, las convenciones internacionales sobre la gestión sostenible de los bosques, la protección de la biodiversidad y la lucha contra la desertificación, el enfoque de la gestión forestal ha cambiado hacia una visión multifuncional del bosque. Desde 2008 la Dirección General de Bosques ha adoptado los principios de uso múltiple y de gestión forestal sostenible en Turquía. La nueva filosofía de gestión tiene cuatro pilares importantes; integración de la conservación de la biodiversidad en el proceso de gestión forestal, la caracterización y el mantenimiento de la multifuncionalidad de los bosques, la participación efectiva de los interesados y el uso de tecnologías de la información y de nuevas técnicas de gestión.

El pino silvestre (Pinus sylvestris L.) es una de las principales especies forestales en Turquía. En este país está presente principalmente en el norte y noreste y cuenta con una superficie aproximada de 1,5 millones de hectáreas y una estimación de volumen alrededor de $120 \mathrm{Mm}^{3}$ de madera (General Directorate of Forestry-Turkey 2012). Crece en zonas con precipitación anual diversa (desde una precipitación total anual de $360 \mathrm{~mm}$ hasta superior a $2.500 \mathrm{~mm}$ ). Esta especie se encuentra en un rango de altitud de 1.000-2.500 m sobre el nivel del mar, con las excepciones de Sürmene y Sarıkamış, donde se encuentra en altitudes de $10-15 \mathrm{~m}$ y de $2.700 \mathrm{~m}$, respectivamente. La madera de este pino tiene buena resistencia a agentes patógenos y atmosféricos y es una importante fuente de materia prima para la industria forestal en Turquía (aserrado y trituración) y para su uso como leña (Bozkurt y Göker 1996). Además, los bosques de pino silvestre desempeñan un papel clave en el suministro de importantes beneficios sociales y servicios ambientales como la protección de los recursos suelo y agua y la conservación de la diversidad biológica en el norte del país.

Debido al interés maderero del pino silvestre se hace necesaria la estimación del volumen. En Turquía, por lo general, se utilizan tarifas de cubicación locales para estimar el volumen de árbol individual. Estas tarifas relacionan el volumen total con el diámetro y se desarrollan para cada especie a partir de una muestra representativa de datos obtenida de la corta de árboles. Sin embargo, en muchos casos resulta necesario conocer, además del volumen total de madera, qué parte de dicho volumen puede dirigirse a los diferentes destinos industriales (desenrollo, aserrado, trituración, etc.). Las tarifas de cubicación con clasificación de productos clasifican el volumen de madera por destinos y permiten realizar una valoración económica de la producción de una masa más exacta que la proporcionada por las tarifas clásicas, que solamente determinan el valor total del volumen (Diéguez-Aranda et al. 2009). Las funciones de perfil o ahusamiento son la alternativa más habitual para desarrollar tarifas de cubicación con clasificación de productos. Se basan en el ajuste de una ecuación que define la variación del diámetro a lo largo del tronco del árbol y, por tanto, caracteriza su forma (Kozak 2004). La integración de la función de perfil desde el suelo hasta cualquier altura proporciona una estimación del volumen maderable hasta dicha altura. Las funciones de perfil no tienen un amplio uso en Turquía y diferentes ecuaciones estándar de volumen desarrolladas hace más de cinco décadas (e.g. Alemdağ 1967) siguen siendo la herramienta más común que se utiliza para estimar el volumen de las especies arbóreas. Aunque recientemente se han desarrollado varias funciones de perfil para Turquía (e.g. Sakıcı et al. 2008, Özçelik y Brooks 2012), no se ha incluido el pino silvestre. Por lo tanto, se hace necesario el desarrollo de una función de perfil para pino silvestre en Turquía.

Como funciones de perfil se pueden emplear modelos simples, segmentados (e.g. Max y Burkhart 1976) o de exponente variable (e.g. Kozak 2004). Los modelos segmentados fueron introducidos por Max y Burkhart (1976) para obtener una mejor descripción del perfil del tronco que la proporcionada por modelos simples, especialmente en la región basal, que es en la que se acumula más volumen, y han mostrado buenos resultados en estudios previos (Diéguez-Aranda et al. 2006, Trincado y Burkhart 2006, Sabatia y Burkhart 2015). Por lo general, se asume que un tronco de un árbol puede dividirse en tres zonas geométricas: la zona basal, que se asemeja a un tronco de neiloide, la sección central, que se asemeja a un tronco de paraboloide, y la sección superior, que se asemeja a un cono. Los modelos segmentados describen cada una de estas zonas ajustando una ecuación diferente, para posteriormente unirlas matemáticamente y obtener una función general segmentada.

Los datos requeridos para el ajuste de funciones de perfil son en su mayor parte jerárquicos, con múltiples medidas de diámetro a lo largo del tronco para cada árbol (Leites y Robinson 2004). Por lo tanto, las observaciones dentro de cada individuo están correlacionadas y dicha correlación dependerá de la distancia entre las mediciones (Tasissa y Burkhart 1998). En estos casos, cuando se ajusta un modelo, también existe correlación entre los residuos de un mismo individuo (autocorrelación), violando la hipótesis de independencia de residuos requerida en la regresión por mínimos cuadrados ordinarios. Una consecuencia de esta autocorrelación es que las varianzas de las estimaciones de los parámetros son sesgadas e inconsistentes, invalidando los contrastes de significación, aunque tales estimaciones de los parámetros no se vean afectadas (West et al. 1984). 
Aunque existen varias técnicas para analizar conjuntos de datos con una estructura jerárquica, una de las más utilizadas actualmente es la de modelos mixtos. Numerosos estudios han utilizado modelos mixtos para desarrollar funciones de perfil (e.g. Tasissa y Burkhart 1998, Trincado y Burkhart 2006, Gómez-García et al. 2013, Arias-Rodil et al. 2015).

Un modelo mixto puede proporcionar una respuesta media, si se consideran solamente los parámetros de efectos fijos (para simplificar, de aquí en adelante se denominarán parámetros fijos), que son comunes a toda la población, mientras que permite obtener una respuesta específica por individuo si se añaden efectos aleatorios a los parámetros fijos, que en conjunto forman los denominados parámetros de efectos mixtos (de aquí en adelante parámetros mixtos). Los modelos mixtos explican directamente la variación en la forma del tronco dentro de un árbol y entre diferentes árboles (Davidian y Giltinan 1995). En la fase de aplicación, si se dispone de mediciones complementarias de la variable respuesta (en este caso el diámetro del tronco a una determinada altura) se pueden estimar los efectos aleatorios. Este proceso se denomina calibración y la respuesta que genera, respuesta calibrada. El empleo de modelos mixtos y la predicción de los efectos aleatorios a partir de mediciones de diámetro a diferentes alturas han sido utilizados con éxito por varios autores (e.g. Trincado y Burkhart 2006, Yang et al. 2009, Gómez-García et al. 2013). Definir qué parámetros deben ser mixtos es clave en el desarrollo de estos modelos. La mayoría de estudios basan su decisión en los estadísticos de bondad de ajuste. Sin embargo, GómezGarcía et al. (2013) desarrollaron una función de perfil para Betula pubescens Ehrh. en España, y observaron que el mejor modelo mixto en la fase de ajuste no era el que mejor se comportaba en la fase de calibración. Estos autores concluyeron evaluar otras especies y otros modelos diferentes al de exponente variable de Kozak (2004) que ellos analizaron.

Los objetivos de este estudio son: (1) desarrollar una función de perfil empleando la metodología de modelos mixtos para Pinus sylvestris en Turquía, analizando qué parámetro o parámetros deberían ser mixtos, y (2) evaluar la respuesta calibrada a partir de mediciones del diámetro a diferentes alturas. Las hipótesis del trabajo son: (1) existe una correlación entre los residuos de un mismo individuo (autocorrelación), por lo que es necesario emplear la metodología de modelos mixtos, y (2) la calibración del modelo mixto mediante mediciones complementarias de la variable respuesta mejora la respuesta media del modelo mixto (empleando solamente los parámetros de efectos fijos).

\section{MÉTODOS}

Datos. Los datos empleados en este estudio proceden del muestreo destructivo de 100 árboles talados en bosques naturales de $P$. sylvestris en Turquía. Se cortaron árboles de diferentes clases sociológicas, que no presentaban malformaciones en el tronco y la copa, y sin desarrollo anómalo o desequilibrios motivados por su proximidad a claros o bordes de masa o pista. Antes de cortarlos se realizaron en cada uno de ellos dos mediciones perpendiculares del diámetro normal $(d$, diámetro a $1,3 \mathrm{~m}$ sobre el suelo desde la parte ladera arriba del árbol) con una precisión de 0,25 $\mathrm{cm}$. Una vez derribados los árboles, se midió con cinta métrica la longitud total del tronco $(h)$ con aproximación al decímetro. Posteriormente, el tronco se dividió en trozas de $1 \mathrm{~m}$ y, en los extremos se realizaron dos mediciones perpendiculares del diámetro con corteza $\left(d_{i}\right)$ mediante una forcípula de precisión milimétrica.

Para examinar si existían anomalías en los datos se representó y analizó visualmente el gráfico de diámetros relativos frente a alturas relativas (figura 1), que son respectivamente el cociente entre el diámetro $d_{i}$ a la altura $h_{i}$ y el diámetro normal $d$, y el cociente entre $h_{i}$ y la altura total del árbol $h$. No se observaron datos atípicos, por lo que se emplearon los datos originales en el desarrollo de la función de perfil (cuadro 1).

Ajuste de modelos y criterios de selección. En un análisis previo se ajustaron mediante regresión no lineal diferentes funciones de perfil que habían resultado exitosas en estudios previos. El mejor fue el modelo segmentado de Max y Burkhart (1976) (ecuación 1), que ha demostrado buenos resultados en numerosos estudios y una gran flexibilidad de adaptación a diferentes especies y tipos de masas (e.g. Trincado y Burkhart 2006, Cao 2009, Sabatia y Burkhart 2015).

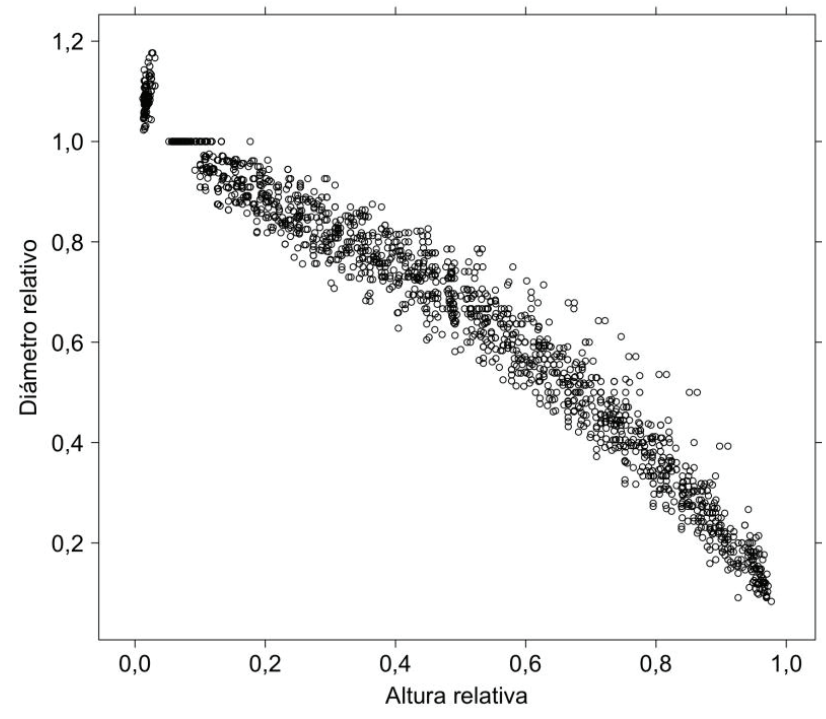

Figura 1. Diámetro relativo $(d / d)$ frente a altura relativa $(h / h)$ de los árboles empleados en el ajuste.

Relative diameter $(d / d)$ against relative height $(h / h)$ of trees used in the fitting step. 
Cuadro 1. Estadísticos descriptivos de los datos empleados en el ajuste de la función de perfil para $P$. sylvestris en Turquía. Summary statistics of the data set used for fitting the stem taper function for P. sylvestris in Turkey.

\begin{tabular}{lcccc}
\hline \multicolumn{1}{c}{ Variable } & Media & Mínimo & Máximo & Desviación estándar \\
\hline $\mathrm{N}^{\circ}$ secciones por árbol & 17 & 10 & 25 & 3,6 \\
$d(\mathrm{~cm})$ & 30,1 & 15,0 & 44,0 & 7,4 \\
$h(\mathrm{~cm})$ & 17,8 & 9,8 & 25,0 & 3,6 \\
\hline
\end{tabular}

Nota: $d$ = diámetro normal con corteza (a 1,3 m sobre el nivel del suelo), $h=$ altura total del árbol.

$\left(\frac{d_{i}}{d}\right)^{2}=b_{1}(q-1)+b_{2}\left(q^{2}-1\right)+b_{3}\left(a_{1}-q\right)^{2} I_{1}+b_{4}\left(a_{2}-q\right)^{2} I_{2}+\varepsilon[1]$

$\begin{array}{ll}\text { donde } & I_{1}=1 \text { si } q \leq a_{1}, 0 \text { en caso contrario } \\ I_{2}=1 \text { si } q \leq a_{2}, 0 \text { en caso contrario }\end{array} \quad q=h_{i} / h$

Nota: $d_{i}=$ diámetro con corteza $(\mathrm{cm})$ a la altura $h_{i}(\mathrm{~m}) ; d$ = diámetro normal con corteza $(1,3 \mathrm{~m}$ sobre el suelo, $\mathrm{cm})$; $h=$ altura total del árbol $(\mathrm{m}) ; b_{1}, b_{2}, b_{3}, b_{4}, a_{1}$ y $a_{2}=$ parámetros a estimar; $\varepsilon=$ término de error.

Empleando modelos mixtos sobre funciones no lineales, la función de perfil puede expresarse, de forma general, como:

$$
\mathrm{d}_{t}=f\left(\mathrm{x}_{t}, \varphi_{t}\right)+\varepsilon_{t}, \quad \varepsilon_{t} \sim \mathrm{N}\left(0, \mathrm{R}_{t}\right)
$$

donde:

$\mathbf{d}_{t}=$ vector $\left(n_{t} \times 1\right)$ de diámetros con corteza observados para un árbol $t$.

$f()=$. función no lineal.

$\mathbf{x}_{t}=$ matriz $\left(n_{t} \mathrm{x} s_{1}\right)$ conocida de variables

$\boldsymbol{\varphi}_{t}=$ vector de parámetros $\left(s_{1} \times 1\right)$, que puede estar compuesto sólo por los parámetros fijos o por los parámetros fijos más los efectos aleatorios.

$\boldsymbol{\varepsilon}_{t}=$ vector $\left(n_{t} \times 1\right)$ del término de error.

$\mathbf{R}_{t}=$ matriz $\left(n_{t} \times n_{t}\right)$ definida positiva de varianzas-covarianzas del término de error.

El vector de parámetros $\boldsymbol{\varphi}_{t}$ puede expresarse como (Lindstrom y Bates 1990):

$$
\varphi_{t}=A_{t} \beta+B_{t} u_{t}, \quad u_{t} \sim N(0, D)
$$

donde:

$\boldsymbol{\beta}=$ vector $\left(s_{1} \times 1\right)$ de parámetros fijos comunes para todos los árboles.

$\mathbf{u}_{t}=$ vector $\left(s_{2} \times 1\right)$ de efectos aleatorios asociados a un árbol $t$, que se asume que siguen una distribución normal multivariante de media 0 y matriz de varianzascovarianzas D.

$\mathbf{A}_{t}=$ matriz de diseño para los parámetros fijos.

$\mathbf{B}_{t}=$ matriz de diseño para los efectos aleatorios.
Las matrices $\mathbf{A}_{t}$ y $\mathbf{B}_{t}$ contienen usualmente, pero no necesariamente, sólo ceros y unos como elementos (Fang y Bailey 2001). Una situación común es que solo algunos de los parámetros del modelo sean mixtos (parámetros fijos que tienen asociados efectos aleatorios). $\mathbf{A}_{t}$ es una matriz identidad de tamaño $\left(s_{1} \times s_{1}\right)$ y $\mathbf{B}_{t}$ es una matriz de tamaño $\left(s_{2} \times s_{2}\right)$ que contiene solo un subconjunto de las columnas de $\mathbf{A}_{t}$ (Lindstrom y Bates 1990), correspondiendo a aquellos parámetros fijos donde se añadieron efectos aleatorios. Así, la ecuación 2 puede expresarse del siguiente modo:

$\mathrm{d}_{t}=f\left(\mathrm{x}_{t}, \varphi_{t}\right)+\varepsilon_{t}=f\left(\mathrm{x}_{t}, \beta, \mathrm{u}_{t}\right)+\varepsilon_{t}, \varepsilon_{t} \sim \mathrm{N}\left(0, \mathrm{R}_{t}\right)[4]$

Por lo tanto, se debe decidir como será la matriz $\mathbf{B}_{t}$, es decir, qué parámetros serán mixtos, i.e. qué parámetros fijos tendrán asociados efectos aleatorios. Para tomar esta decisión, en este estudio se ajustaron modelos que consideraban diferentes combinaciones de parámetros mixtos. Se analizaron todas las posibles combinaciones con uno y dos parámetros mixtos y para determinar cuál era la mejor en la fase de ajuste se calculó el AIC y el BIC (criterio de información de Akaike y criterio de información Bayesiano de Schwarz, respectivamente). También se probó una alternativa a este procedimiento, propuesta por Fang y Bailey (2001), que consiste en realizar un ajuste independiente para cada individuo, mediante regresión no lineal, del modelo de parámetros fijos, es decir, sin considerar efectos aleatorios y expandir con estos los parámetros que presenten mayor variabilidad. Esta aproximación requiere disponer de suficientes observaciones de cada individuo para que los parámetros estimados sean significativos en cada ajuste individual; en caso contrario, puede trabajarse con grupos de individuos. El coeficiente de variación se empleó para cuantificar la variabilidad de cada parámetro en los diferentes ajustes.

Algunos estudios con funciones de perfil han indicado que la autocorrelación puede reducirse prácticamente en su totalidad con la inclusión de efectos aleatorios (e.g. Yang et al. 2009). Sin embargo, en otros trabajos (e.g. Trincado y Burkhart 2006, Gómez-García et al. 2013), el uso de dichos efectos solo permitió reducir parcialmente la autocorrelación. Incluso en el trabajo de Arias-Rodil et al. (2015) se observó que ciertas combinaciones de parámetros mixtos no mostraban autocorrelación, mientras que otras sí. En este 
estudio, cuando fue necesario debido a la presencia de autocorrelación, se modificó la matriz de varianzas-covarianzas del término de error empleando una estructura autorregresiva $A R(1)$, aplicable cuando las observaciones de diámetro a lo largo del tronco son equidistantes. En este caso, la matriz $\mathbf{R}_{t}$ se modeliza como $\sigma^{2} \times \Gamma_{i}(\rho)$, donde $\sigma^{2}$ es la varianza del error y $\Gamma_{i}(\rho)$ es una matriz $\left(n_{t} \times n_{t}\right)$ que describe el patrón de correlación entre las mediciones del individuo $t$. Para el elemento $i j$-ésimo la estructura de covarianza es $\rho^{|i-j|}$.

En el ajuste del modelo mixto se empleó la función nlme del paquete nlme (Pinheiro et al. 2013) del programa de análisis estadístico R (R Core Team 2015), que estima los parámetros fijos, la matriz de covarianzas de los efectos aleatorios y la varianza del error. El ajuste se realiza linealizando el modelo no lineal mediante una expansión en series de Taylor de primer orden. Esta expansión se puede realizar en torno a cero o en torno al mejor predictor empírico lineal no sesgado (EBLUP) (Lindstrom y Bates 1990). Yang y Huang (2013) los designaron como métodos FO (first-order) y FOCE (first-order conditional expectation), respectivamente, y observaron que el empleo del primero puede proporcionar predicciones biológicamente no razonables en el proceso de calibración, por lo que en este estudio se empleó el método FOCE o de expansión en torno a EBLUP.

El ajuste del modelo se puede realizar tanto por el procedimiento de máxima verosimilitud (ML) como el de máxima verosimilitud restringida (REML). El primero proporciona valores de máxima verosimilitud resultantes comparables entre modelos (en contra de lo que pasa con el procedimiento REML), por lo que los estadísticos de comparación AIC y BIC se basan en dichos valores. Sin embargo, las estimaciones con ML de los componentes de la varianza no tienen en cuenta los grados de libertad que se pierden en la estimación de los parámetros de efectos fijos y por tanto son sesgados a la baja. Por su parte, las estimaciones REML son insesgadas (Laird y Ware 1982), por lo que el ajuste final de los modelos se realizó con este procedimiento.

Calibración del modelo mixto. Una vez ajustada la función de perfil mediante un modelo mixto, es posible predecir una respuesta media de los diámetros a diferentes alturas del tronco para un nuevo individuo $t$. Esta respuesta media se obtiene al emplear el modelo mixto considerando el valor de los efectos aleatorios igual a cero:

$$
\widehat{\mathrm{d}}_{t}=f\left(\mathrm{x}_{t}, \widehat{\beta}, 0\right)
$$

En cambio, si se dispone de una o más mediciones del diámetro a diferentes alturas del tronco, el modelo mixto se puede calibrar, es decir, se pueden estimar los efectos aleatorios para un nuevo individuo $t$. La estimación de efectos aleatorios se realiza mediante el cálculo de un estimador Bayesiano aproximado (Vonesh y Chinchilli 1997): donde $\mathbf{Z}_{t}$ es la matriz de derivadas parciales con respecto a $\operatorname{los}$ efectos mixtos $\mathbf{Z}_{t}=\partial f\left(\mathbf{x}_{t}, \boldsymbol{\beta}, \mathbf{u}_{t}\right) /\left.\partial u_{t}\right|_{\hat{\beta}, \hat{\mathbf{u}}_{*}}$.

Una vez estimados los efectos aleatorios para cada nuevo individuo, se puede estimar el diámetro con corteza a diferentes alturas utilizando un modelo calibrado específico (Vonesh y Chinchilli 1997):

$$
\widehat{\mathrm{d}}_{t}=f\left(\mathrm{x}_{t}, \widehat{\beta}, \hat{\mathrm{u}}_{t}\right)
$$

En este trabajo se evaluó cuál era la mejor altura a la que medir un diámetro adicional al diámetro normal para realizar la calibración. La selección de la mejor alternativa de calibración se basó en el error medio cuadrático (EMC):

$$
\mathrm{EMC}=\frac{\sum_{i=1}^{i=n}\left(Y_{i}-\hat{Y}_{i}\right)^{2}}{n-p}
$$

donde

$Y_{i}, \hat{Y}_{i}=$ diámetro observado y estimado, respectivamente, para la observación $i$-ésima $(i=1,2, \ldots, n)$.

$n=$ número total de observaciones.

$p=$ número de parámetros del modelo.

Para calcular el EMC, los datos para la calibración se clasificaron según la altura relativa a la que pertenecían. Todas las combinaciones de parámetros fijos a expandir se compararon en el ajuste y la calibración. También se comparó la respuesta calibrada con la proporcionada por un modelo de efectos fijos basado en la ecuación 1, ajustado por mínimos cuadrados ordinarios (OLS, por sus siglas en inglés) con la función $n l s$ de R (R Core Team 2015).

\section{RESULTADOS}

Ajustando todas las posibles combinaciones con uno y dos parámetros mixtos, se comprobó que algunos modelos mixtos no convergían o que presentaban algún parámetro fijo o algún componente de la varianza de los efectos aleatorios no significativo. La combinación que mejores estadísticos de ajuste proporcionó fue considerando mixtos los parámetros $b_{2}$ y $b_{4}$ (cuadro 2). Por otra parte, todos los modelos de efectos mixtos proporcionaron una mejor respuesta que el modelo de efectos fijos.

En el estudio del procedimiento alternativo, que consiste en evaluar la variabilidad de los parámetros a partir de ajustes locales por árboles o grupos de árboles, no se obtuvo convergencia al realizar un ajuste por regresión no lineal para cada árbol. Por lo tanto, se realizaron ajustes independientes para grupos de 10 árboles seleccionados aleatoriamente. Los parámetros $a_{1}, b_{3}$ y $b_{4}$ presentaron mayor variabilidad (cuadro 3 ), con valores del coeficiente de variación del 28, 41 y $28 \%$, respectivamente. Estos resultados no coinciden con los anteriores, donde $a_{1}$ y $b_{3}$ no apa-

$$
\hat{\mathrm{u}}_{t} \cong \widehat{\mathrm{D}} \mathrm{Z}_{t}^{\mathrm{T}}\left(\mathrm{Z}_{t} \widehat{\mathrm{D}} \mathrm{Z}_{t}^{\mathrm{T}}+\widehat{\mathrm{R}}_{t}\right)^{-1} \hat{e}_{k}=\widehat{\mathrm{D}} \mathrm{Z}_{t}^{\mathrm{T}}\left(\mathrm{Z}_{t} \widehat{\mathrm{D}} \mathrm{Z}_{t}^{\mathrm{T}}+\widehat{\mathrm{R}}_{t}\right)^{-1}\left(\mathrm{~d}_{t}-f\left(\mathrm{x}_{t}, \widehat{\beta}, \hat{\mathrm{u}}_{t}\right)+\mathrm{Z}_{t} \hat{\mathrm{u}}_{t}\right)
$$


Cuadro 2. AIC y BIC del modelo de efectos fijos (OLS) y de los modelos mixtos con todos los parámetros fijos y la varianzacovarianza de los efectos aleatorios significativamente distintos de cero a un nivel de significación del $5 \%$ (ordenados de menor a mayor valor del AIC), ajustados para $P$. sylvestris en Turquía.

AIC and BIC of fixed-effects model (OLS) and of mixedeffects models with all fixed parameters and random-effects variancecovariance significant (at a level of 5\%) (sorted by decreasing order of AIC), fitted for $P$. sylvestris in Turkey.

\begin{tabular}{ccc}
\hline Parámetros mixtos & AIC & BIC \\
\hline$b_{2}, b_{4}$ & 3.242 & 3.302 \\
$b_{1}, b_{2}$ & 3.243 & 3.303 \\
$a_{2}, b_{2}$ & 3.245 & 3.305 \\
$b_{1}, b_{4}$ & 3.246 & 3.305 \\
$b_{2}$ & 3.264 & 3.313 \\
$a_{1}, b_{2}$ & 3.266 & 3.326 \\
$b_{2}, b_{3}$ & 3.267 & 3.326 \\
$a_{1}, b_{1}$ & 3.293 & 3.353 \\
$b_{1}$ & 3.294 & 3.343 \\
$b_{1}, b_{3}$ & 3.297 & 3.356 \\
$b_{3}$ & 3.306 & 3.355 \\
$b_{4}$ & 3.306 & 3.355 \\
$a_{2}$ & 3.306 & 3.355 \\
$a_{1}$ & 3.306 & 3.355 \\
$b_{3}, b_{4}$ & 3.309 & 3.369 \\
$a_{2}, b_{4}$ & 3.310 & 3.370 \\
$a_{1}, b_{4}$ & 3.310 & 3.370 \\
$a_{1}, a_{2}$ & 3.310 & 3.370 \\
OLS & 5.750 & 5.788 \\
\hline & &
\end{tabular}

recen como las mejores opciones de parámetros mixtos, tanto considerados individualmente como combinados.

En la fase de calibración, el EMC se redujo a medida que aumentaba la altura de calibración, alcanzando su valor mínimo al $70 \%$ de la altura total del árbol (figura 2). En esta figura no aparecen representados los modelos que consideran mixtos los parámetros $a_{1}, a_{2}, b_{3}$ y $b_{4}$ o las combinaciones $\left(a_{1}, a_{2}\right),\left(a_{1}, b_{4}\right),\left(a_{2}, b_{4}\right)$ y $\left(b_{3}, b_{4}\right)$ debido a que las respuestas calibradas no supusieron mejoras significativas con respecto al modelo de efectos fijos, independientemente de la altura de calibración considerada. Estos modelos mixtos coinciden además con los que presentaron peores resultados en la fase de ajuste. Las respuestas calibradas de los modelos mixtos restantes proporcionaron mejores resultados que el modelo de efectos fijos (figura 2), especialmente para la altura de calibración mencionada ( $70 \%$ de la altura total).
Cuadro 3. Coeficientes de variación de los parámetros obtenidos en el ajuste por regresión no lineal por grupos de 10 árboles para $P$. sylvestris en Turquía.

Coefficients of variation of parameters from non-linear regression fitting by groups of 10 trees of P. sylvestris in Turkey.

\begin{tabular}{cc}
\hline Parámetro & CV (\%) \\
\hline$a_{1}$ & 28 \\
$a_{2}$ & 10 \\
$b_{1}$ & 17 \\
$b_{2}$ & 20 \\
$b_{3}$ & 41 \\
$b_{4}$ & 28 \\
\hline
\end{tabular}

Nota: $\mathrm{CV}=$ coeficiente de variación.

La opción de considerar mixtos los parámetros $b_{2} \mathrm{y}$ $b_{4}$ proporcionó los mejores resultados, tanto en la fase de ajuste como en la de calibración, por lo que se propone como modelo mixto (ecuación 9) para la función de perfil de $P$. sylvestris en Turquía. La respuesta media de este modelo mixto presentó un EMC de 1,900 $\mathrm{cm}^{2}$, mayor que el EMC del modelo OLS $\left(1,774 \mathrm{~cm}^{2}\right)$. $\left(\frac{\hat{d}_{i}}{d}\right)^{2}=b_{l}(q-1)+\left(b_{2}+u_{1}\right)\left(q^{2}-1\right)+b_{3}\left(a_{1}-q\right)^{2} I_{1}+\left(b_{4}+u_{2}\right)\left(a_{2}-q\right)^{2} I_{2}$ [9]

Nota: $u_{1}$ y $u_{2}=$ efectos aleatorios. El resto de variables ya han sido definidas anteriormente.

El gráfico de residuos frente a residuos de una observación previa dentro de cada árbol en el ajuste OLS (figura 3, izquierda) reveló la presencia de autocorrelación. El modelo mixto seleccionado corrigió en parte este problema (figura 3, centro), pero para eliminarla completamente fue necesario emplear una estructura autorregresiva de primer orden AR(1) (figura 3, derecha). Los parámetros del modelo de efectos fijos y del modelo mixto seleccionado (cuadro 4) resultaron todos significativos $(P<0,001)$.

\section{DISCUSIÓN Y CONCLUSIONES}

En este estudio se desarrolla una función de perfil para $P$. sylvestris en Turquía, empleando un modelo mixto en base al modelo segmentado de Max y Burkhart (1976). El empleo de modelos mixtos mejora las estimaciones del modelo de efectos fijos ajustado por OLS. Para seleccionar el modelo mixto final no ha resultado útil estudiar qué parámetros fijos tenían mayor variabilidad al ajustar el modelo por grupos de árboles empleando OLS, ya que los modelos resultantes al aplicar dicho procedimiento fueron claramente inferiores que los mejores obtenidos al ajustar todas las posibles combinaciones con uno y dos parámetros mixtos. Basándose en los resultados obtenidos 


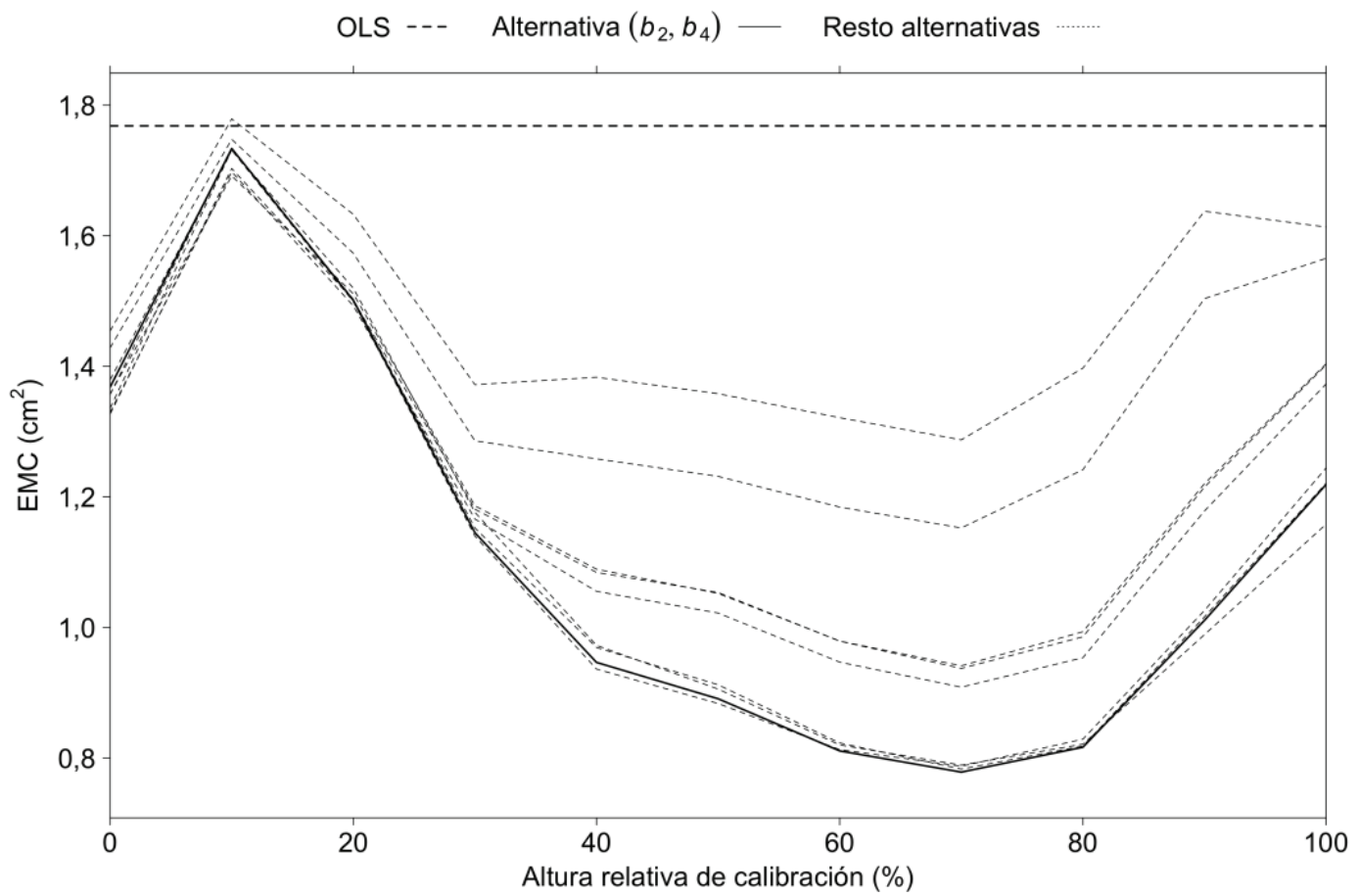

Figura 2. Evolución del EMC con respecto a la altura de calibración para las diferentes combinaciones con uno y dos parámetros mixtos del modelo de Max y Burkhart (1976) para P. sylvestris en Turquía. La respuesta del modelo ajustado por mínimos cuadrados se representa con trazo horizontal y discontinuo, la alternativa seleccionada $\left(b_{2}, b_{4}\right)$ para el modelo de efectos mixtos aparece como un trazo continuo y el resto de alternativas con trazo de puntos.

EMC evolution against calibration height for different combinations of one and two mixed-effects parameters using the model of Max and Burkhart (1976) for P. sylvestris in Turkey. Response of the model fitted by ordinary least-squares is represented by a horizontal dashed line, and the selected combination for mixed-effects model $\left(b_{2}, b_{4}\right)$ corresponds to the solid line, while the remaining combinations appear as dotted lines.



Figura 3. Residuos de diámetro frente a residuos anteriores de la ecuación 1 ajustada por OLS (modelo de efectos fijos, izquierda) y del modelo mixto seleccionado (ecuación 9) sin modelizar el término de error (centro) y considerando una estructura autorregresiva de primer orden (AR(1), derecha).

Diameter residuals against lag-one residuals from Equation 1, fitted by OLS (fixed-effects model, left) and from equation 9, without modelling the error term (middle), and considering a first-order autoregressive structure (AR(1), right). 
Cuadro 4. Estimaciones de los parámetros del modelo de efectos fijos (OLS) y del modelo mixto (ecuación 9) ajustado por el procedimiento de máxima verosimilitud restringida (REML).

Parameter estimates of fixed-effects model (fitted by OLS) and the mixed-effects model (equation 9), fitted by the restricted maximum-likelihood procedure (REML).

\begin{tabular}{llcc}
\hline Parámetros & Parámetro & OLS & Modelo mixto \\
\hline Parámetros fijos & $a_{1}$ & 0,2028 & 0,1754 \\
& $a_{2}$ & 0,7206 & 0,7510 \\
& $b_{1}$ & $-3,097$ & $-3,215$ \\
& $b_{2}$ & 1,377 & 1,413 \\
& $b_{3}$ & 6,084 & 7,228 \\
& $b_{4}$ & $-1,450$ & $-1,403$ \\
\hline Componentes & $\operatorname{var}\left(u_{1}\right)$ & - & 0,005115 \\
& $\operatorname{var}\left(u_{2}\right)$ & - & 0,007480 \\
& $\operatorname{cov}\left(u_{1}, u_{2}\right)$ & - & 0,006167 \\
& $\sigma^{2}$ & - & 0,7360 \\
\hline
\end{tabular}

en este y otros trabajos (e.g. Gómez-García et al. 2013, Arias-Rodil et al. 2015), se recomienda el empleo de modelos mixtos para el ajuste de funciones de perfil ya que permiten considerar la estructura jerárquica característica de los conjuntos de datos empleados para el desarrollo de las mismas.

Se evaluaron los modelos mixtos tanto mediante el análisis de sus ajustes (comparando los estadísticos AIC y BIC) como de sus calibraciones (comparando el estadístico EMC). De un total de 21 modelos mixtos, tres fueron descartados ya que poseían algún parámetro fijo o la varianza-covarianza de algún efecto aleatorio no significativos a un nivel de significación del $5 \%$. El modelo mixto finalmente propuesto es el que considera los parámetros $b_{2}$ y $b_{4}$ como mixtos. Este modelo presenta los estadísticos más favorables tanto en la fase de ajuste como en la de calibración, al contrario que lo observado en el trabajo de Gómez-García et al. (2013) para la especie Betula pubescens. Los resultados obtenidos por estos autores podrían deberse a que emplearon el método FOCE para ajustar el modelo y el método FO para la calibración (Yang y Huang 2013). En el presente estudio, al igual que en otro previamente realizado por Arias-Rodil et al. (2015), se emplea el método FOCE para ambas fases, observándose que el mismo modelo se comporta mejor en los dos casos.

Como era de esperar, se observó la presencia de autocorrelación en el ajuste del modelo de efectos fijos OLS. En estudios previos, como los realizados por Yang et al. (2009) o Arias-Rodil et al. (2015), el empleo de modelos mixtos explicó prácticamente en su totalidad la correlación entre los residuos dentro de un mismo árbol. Sin embargo, en este estudio, al igual que en los de Trincado y Burkhart (2006) o Gómez-García et al. (2013), el empleo de modelos mixtos no es suficiente para eliminar totalmente la autocorrelación entre los residuos, por lo que es necesario modelizar directamente el término de error mediante una estructura autorregresiva de primer orden AR(1).

La respuesta media del modelo mixto (i.e. empleando solamente los parámetros fijos) genera peores resultados que el ajuste con el modelo OLS. Sin embargo, la respuesta calibrada usando un diámetro complementario mejora las estimaciones del modelo OLS, independientemente de la altura a la que se mida ese diámetro. Los mejores resultados se obtienen utilizando diámetros medidos a alturas relativas entre 40 y $90 \%$ de la altura total del árbol. Este resultado es acorde con los obtenidos en estudios previos, los cuales identifican la zona central del tronco como la mejor para medir diámetros complementarios para calibrar (e.g. Cao 2009, Arias-Rodil et al. 2015).

Aunque algunos autores han intentado mejorar las estimaciones de las funciones de perfil incluyendo variables relacionadas con las dimensiones de la copa, la edad del árbol $\mathrm{u}$ otras variables cuantificadas a los niveles individual o de rodal (e.g. Tasissa y Burkhart 1998), la conclusión general es que las mejoras en las estimaciones, en el caso de que las hubiese, eran pequeñas y que el esfuerzo de medir estas variables no era justificable. Sin embargo, la inclusión de efectos aleatorios mediante la aplicación de modelos mixtos es un procedimiento efectivo para capturar la variación que podría ser explicada por esas variables. Por otra parte, en el caso de que no sea posible la medición de un diámetro adicional para la calibración del modelo mixto, se recomienda el empleo del modelo de efectos fijos ajustado por OLS, ya que desde un punto de vista predictivo proporciona resultados más fiables que la respuesta media del modelo mixto.

Las funciones de perfil se pueden integrar para proporcionar estimaciones de volumen hasta cualquier diámetro en punta delgada. Si el objetivo principal es estimar el volumen total del árbol, es decir, si no es necesaria una clasificación previa de los posibles productos en función de un diámetro límite y una longitud de troza dados, es preferible el desarrollo de ecuaciones de volumen independientes o el uso de funciones de perfil que empleen $d^{2}$ en lugar de $d$ como variable dependiente.

\section{AGRADECIMIENTOS}

Los autores agradecen la financiación por parte del Fondo Social Europeo y del gobierno de España a través del INIA y del Ministerio de Educación, Cultura y Deporte (ayuda FPU, AP2012-05337). El grupo de investigación UXFS está parcialmente financiado por la Xunta de Galicia, España, a través del Programa de Consolidación y Estructuración de Unidades de Investigación Competitivas 2014-2015. 


\section{REFERENCIAS}

Alemdağ Ş. 1967. Structure and yield potential of Scots pine ( $P i$ nus sylvestris L.) forests in Turkey and the principles to be followed in managing these forest. Ankara, Turkey. Forestry Research Publication, Technical Bulletin. 160 p.

Arias-Rodil M, U Diéguez-Aranda, F Rodríguez Puerta, CA López-Sánchez, E Canga Líbano, A Cámara Obregón, F Castedo-Dorado. 2015. Modelling and localizing a stem taper function for Pinus radiata in Spain. Canadian Journal of Forest Research 45(6): 647-658.

Bozkurt AY, Y Göker. 1996. Forest products utilization. Istanbul, Turkey. Istanbul University Press. 440 p.

Cao QV. 2009. Calibrating a segmented taper equation with two diameter measurements. Southern Journal of Applied Forestry 33(2): 58-61.

Davidian M, D Giltinan. 1995. Nonlinear models for repeated measurement data. New York, USA. Chapman \& Hall. 360 p.

Diéguez-Aranda U, F Castedo-Dorado, JG Álvarez-González, A Rojo. 2006. Compatible taper function for Scots pine plantations in northwestern Spain. Canadian Journal of Forest Research 36(5): 1190-1205.

Diéguez-Aranda U, A Rojo Alboreca, F Castedo-Dorado, JG Álvarez González, M Barrio-Anta, F Crecente-Campo, JM González González, C Pérez-Cruzado, R Rodríguez Soalleiro, CA López-Sánchez, MA Balboa-Murias, JJ Gorgoso Varela, F Sánchez Rodríguez. 2009. Herramientas selvícolas para la gestión forestal sostenible en Galicia. Santiago de Compostela, España. Xunta de Galicia. 259 p.

Fang Z, RL Bailey. 2001. Nonlinear mixed effects modeling for slash pine dominant height growth following intensive silvicultural treatments. Forest Science 47(3): 287-300.

General Directorate of Forestry. 2012. Forest Resources. Ankara, Turkey. The General Directorate of Forest. 24 p.

Gómez-García E, F Crecente-Campo, U Diéguez-Aranda. 2013. Selection of mixed-effects parameters in a variable exponent taper equation for birch trees in northwestern Spain. Annals of Forest Science 70(7): 707-715.

Kozak A. 2004. My last words on taper functions. Forestry Chronicle 80(4): 507-515.

Laird NM, JH Ware. 1982. Random-effects models for longitudinal data. Biometrics 38(4): 963-974.

Leites LP, AP Robinson. 2004. Improving taper equations of lo- blolly pine with crown dimensions in a mixed-effects modelling framework. Forest Science 50(2): 204-212.

Lindstrom MJ, DM Bates. 1990. Nonlinear mixed-effects models for repeated measures data. Biometrics 46(3): 673-687.

Max TA, HE Burkhart. 1976. Segmented polynomial regression applied to taper equations. Forest Science 22(3): 283-289.

Özçelik R, JR Brooks. 2012. Compatible volume and taper models for economically important tree species of Turkey. Annals of Forest Science 69(1): 105-118.

Pinheiro JC, DM Bates, S DebRoy, D Sarkar, R Core Team. 2013. Nlme: Linear and Nonlinear Mixed Effects Models. R Package. Vienna, Austria. R Foundation for Statistical Computing.

R Core Team. 2015. R: A Language and Environment for Statistical Computing. Vienna, Austria. R Foundation for Statistical Computing. Consultado 26 feb. 2015. Disponible en http://www.r-project.org.

Sabatia CO, HE Burkhart. 2015. On the use of upper stem diameters to localize a segmented taper equation to new trees. Forest Science 61(3): 411-423.

Sakıc1 OE, N Misır, H Yavuz, M Misır. 2008. Stem taper functions for Abies nordmanniana subsp. bornmulleriana in Turkey. Scandinavian Journal of Forest Research 23(6): 522-533.

Tasissa G, HE Burkhart. 1998. An application of mixed effects analysis to modelling thinning effects on stem profile of loblolly pine. Forest Ecology and Management 103(1): 87-101.

Trincado G, HE Burkhart. 2006. A generalized approach for modelling and localizing stem profile curves. Forest Science 52(6): 670-682.

Vonesh EF, VM Chinchilli. 1997. Linear and nonlinear models for the analysis of repeated measurements. New York, USA. Marcel Dekker. $560 \mathrm{p}$.

West PW, DA Ratkowsky, AW Davis. 1984. Problems of hypothesis testing of regressions with multiple measurements from individual sampling units. Forest Ecology and Management 7(3): 207-224.

Yang Y, S Huang, G Trincado, SX Meng. 2009. Nonlinear mixedeffects modeling of variable-exponent taper equations for lodgepole pine in Alberta, Canada. European Journal of Forest Research 128(4): 415-429.

Yang Y, S Huang. 2013. On the statistical and biological behaviors of nonlinear mixed forest models. European Journal of Forest Research 132(5-6): 727-736. 
